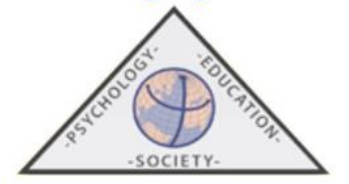

\title{
Diversidad étnico-cultural, inteligencia emocional y competencias socioemocionales en la educación secundaria andaluza
}

\author{
Vicente J. Llorent, Izabela Zych, María Fontans y Mercedes Álamo \\ Universidad de Córdoba, España \\ Recibido el 22 de agosto de 2020. Aceptado el 18 de abril de 2021
}

RESUMEN: La diversidad étnico-cultural es una realidad que está presente en los centros educativos, y debe ser analizada y estudiada en profundidad. En esta realidad se generan grupos de mayorías y minorías, donde estos últimos suelen ser grupos vulnerables dadas sus características sociales y económicas. La evidencia científica avala que la inteligencia emocional y las competencias socioemocionales son factores protectores para el desarrollo del individuo, razón por la cual es necesario estudiar ambos factores teniendo en cuenta la diversidad étnico-cultural. El presente estudio pretende describir la diversidad étnico-cultural, la inteligencia emocional y las competencias socioemocionales en la Educación Secundaria en Andalucía. Para ello, se ha obtenido una muestra representativa de la Educación Secundaria Obligatoria en Andalucía (España), compuesta por 2,139 discentes. Como instrumento de recogida de datos se ha empleado la encuesta, empleando la versión española de la escala Trait Meta-Mood (TMMS) y el Cuestionario de Competencias Sociales y Emocionales (SEC-Q). Los resultados evidencian la presencia heterogénea en cuanto a diversidad étnico-cultural en la escuela andaluza con una mayor presencia de inmigrantes en las escuelas de la provincia de Almería. La inteligencia emocional percibida no presenta diferencias entre los grupos étnicos-culturales. Por el contrario, sí existen diferencias entre los grupos étnicos-culturales en el nivel de competencias socioemocionales del alumnado, concretamente en la autoconciencia. Esto revela la necesidad de promoción de dichas competencias en los escolares, teniendo en cuenta la diversidad del aula, apoyando la necesidad de impulsar la educación inclusiva.

Palabras clave: Diversidad étnico-cultural; inteligencia emocional; competencias socioemocionales; educación secundaria; educación inclusiva.

\section{Ethnic-cultural diversity, emotional intelligence and social and emotional competencies in andalusian secondary education}

\begin{abstract}
Ethnic-cultural diversity is a reality that is present in schools and it needs to be thoroughly analyzed and studied. In this reality, groups of majorities and minorities are generated, where the minorities are usually vulnerable groups given their social and economic characteristics. Scientific evidence supports that emotional intelligence and social and emotional competencies are protective factors for the development of the individuals, which is why it is necessary to study both factors in these most vulnerable groups. The current study describes the ethnic-cultural diversity, the level of emotional intelligence and social and emotional competencies in Andalusian schools. This study included a representative sample of 2139 Andalusian students of Secondary Education. The Spanish version of the Trait Meta-Mood scale (TMMS) and the Social and Emotional Competencies Questionnaire (SEC-Q) have been used. The results showed that the Andalusian schools are diverse in terms of ethnic-cultural diversity, and there was a greater presence of immigrants in the province of Almeria. There were no differences in perceived emotional intelligence among different ethnic-cultural groups. Nevertheless, there were differences among ethnic-cultural groups in the level of social and emotional competencies of the students, specifically in selfawareness. This reveals the need to promote these skills in students, considering the diversity of the classroom, supporting the need to promote inclusive education.

Keywords: Ethnic-cultural diversity; emotional intelligence; social and emotional competencies; secondary education; inclusive education.
\end{abstract}

Correspondencia: Mercedes Álamo. Departamento de Educación, Universidad de Córdoba. Calle Medina Azahara s/n, 14071, Córdoba, España. E-mail: mmalamo@uco.es 


\section{Introducción}

La diversidad étnico-cultural se hace cada vez más visible a través de los continuos movimientos migratorios. En España ha aumentado en el año 2019 un 3.8\% el número de personas extranjeras que residen en el país con respecto al año anterior, llegando a un total de 5,023,279 de personas, según fuentes del Instituto Nacional de Estadística (2020). El mayor número de población residente extrajera viene de Marruecos y Rumanía, si bien el crecimiento más destacado en 2019 tiene su origen en personas de nacionalidad colombiana y venezolana (INE, 2020). Esta realidad social también está presente en las escuelas españolas. Así mismo, en el curso escolar 2017-2018, se ha matriculado en la etapa de la Educación Secundaria Obligatoria 167,229 alumnado extranjero. Andalucía es unas de las comunidades autónomas con mayor número de inmigrantes (INE, 2007, 2020) y el porcentaje de alumnos extranjeros en Andalucía sobre el total de alumnos del país es de un 5.3\%. En esta misma comunidad, en centros de titularidad pública, se ha matriculado el $82.9 \%$ del alumnado extranjero en el curso escolar 2016-2017 mientras que el $7.9 \%$ se ha matriculado en centros concertados y el $9.2 \%$ en centros de titularidad privada no concertada (Ministerio de Educación y Formación profesional, 2018).

La enseñanza de la cultura, valores y creencias de los inmigrantes son trasmitidas a sus futuras generaciones a través de la educación familiar. Así pues, los descendientes de inmigrantes, sea cual sea el país en el que hayan nacido, mantendrán normas de su cultura y costumbres familiares, que además se incluirán en la construcción de su identidad cultural (Llorent-Bedmar y Terrón-Caro, 2013). Según un estudio de Portes et al. (2009), las segundas generaciones de inmigrantes dan más valor a la identidad nacional que a la identidad religiosa, identificándose con la nacionalidad de procedencia familiar. No obstante, los inmigrantes de segunda generación presentan diferencias con respecto a los inmigrantes de primera generación. Como señalan Checa y Monserrat (2015), los estudiantes inmigrantes de segunda generación tienden a acercarse y asimilar en mayor grado las normas y cultura de grupos mayoritarios, a diferencia de los inmigrantes de primera generación. Por tanto, en un primer momento, las personas adquieren una identidad étnico-cultural de sus familias antes de llegar a la escuela, que después puede verse modificada por otros factores.

La diversidad étnico-cultural no solo engloba a inmigrantes, sino también a otros grupos minoritarios como son las personas de etnia gitana. El aumento de la escolarización de las personas de etnia gitana ha sido posible gracias a una serie de medidas que han permitido facilitar su acceso a la escuela (Garreta y Llevot, 2007). Se estima que Andalucía es una de las comunidades con más residentes de etnia gitana (Fundación de Secretariado Gitano, 2009). Por estas razones, la existencia de la diversidad étnico-cultural en los centros andaluces es evidente, y su proporción debe ser estudiada y contabilizada (Llorent et al., 2016; Llorent y González-Gómez, 2020). Por ello, hace falta un estudio descriptivo que aporte datos recientes sobre la diversidad étnico-cultural en las escuelas andaluzas.

La escuela se convierte en un punto de encuentro y convivencia intergrupal, pues es uno de los contextos en el que más interacción se da entre grupos de diferentes países de origen (Aguaded, 2006). Así pues, las escuelas son clave para desarrollar e impulsar una educación que vele por la inclusión de todo el alumnado, incluyendo las minorías étnicas-culturales (Portes y Rumbaut, 2001). En este sentido, ser conscientes y describir la diversidad étnico-cultural de las aulas y su papel socioeducativo podría influir positivamente en la práctica escolar.

Además, las minorías pueden ser colectivos vulnerables, pues Rodríguez-Hidalgo et al. (2014) encontraron que su nivel de autoestima es menor que el del grupo mayoritario, y además pueden estar más implicados en el acoso y ciberacoso (Llorent et al., 2016). Algunos estudios muestran que pueden sufrir victimización racista (Reichelmann et al., 2020; Rodríguez-Hidalgo et al., 2019; Zych y Llorent, 2020). Estas variables son claves para la mejora de las relaciones sociales entre los estudiantes en 
contextos de diversidad étnico-cultural, lo cual permitirá la construcción de la educación inclusiva en la escuela.

\section{Inteligencia emocional}

La inteligencia emocional (IE) podría facilitar la inclusión y el ajuste social y psicológico (Beltrán-Catalán, et al., 2018), en cuanto que es una habilidad por la que se reconocen, comprenden y manejan los estados de ánimo, con un beneficio hacia uno mismo y hacia los demás (Mayer et al., 2004; Salovey y Grewal, 2005). Además, es un factor que influye en el crecimiento personal y en el ajuste emocional (Fernández-Berrocal y Ramos, 2005). La IE permite percibir, comprender, utilizar y gestionar las emociones en uno mismo y hacia los demás (Mayer y Salovey, 1997). Así pues, una persona emocionalmente inteligente es capaz de responder a las emociones que se le presentan, puede comprender las causas y consecuencias de éstas y desarrollar estrategias para manejar dichas emociones.

La IE percibida es la percepción que tiene la persona sobre su propio nivel de inteligencia emocional. Según el modelo de Fernández-Berrocal y Ramos (2005), basado en Salovey et al. (1995), esta percepción sobre la propia IE está formada por tres componentes o factores: atención emocional, claridad emocional, y la reparación o regulación emocional.

Quienes son capaces de expresar y entender las emociones, así como reconocerlas en diferentes situaciones y regularlas, tienen un ajuste psicológico y social más saludable (Ciarrochi et al., 2001). La IE percibida y el ajuste psicológico de las personas están asociados a las estrategias de afrontamiento que se ponen en juego, y al pensamiento reflexivo que cada individuo hace sobre sus propias emociones. En concreto, una regulación emocional y un reconocimiento adecuados de las emociones está relacionado con un pensamiento reflexivo (Gohm y Clore, 2002) y un afrontamiento activo (Berking et al., 2008). Por el contrario, una reconsideración y afrontamiento pasivo de las emociones, tiene una relación negativa con respecto al ajuste psicológico y la IE percibida (Ramos et al., 2007; Salovey et al., 2002). Otro estudio señala la relación positiva entre la claridad y regulación emocional percibida y la satisfacción vital de los adolescentes (Sánchez-Álvarez et al., 2015). Sin embargo, estos autores concluyeron que la atención emocional excesiva y prolongada se relaciona con menor satisfacción vital de los adolescentes.

En cuanto a la IE según el origen cultural, los estudios demuestran que existen diferencias entre los grupos étnicos (Dewi et al., 2018). En el estudio de Martines et al. (2006), llevado a cabo en Estados Unidos y México, los resultados mostraron unas puntuaciones significativamente más bajas en atención y comprensión emocional en los grupos de origen mexicano, mientras que las puntuaciones eran más altas en los afroamericanos, latinoamericanos y caucásicos. Otro estudio más reciente evidencia que la cultura actúa como moderadora de la inteligencia emocional, si bien la IE está valorada en todas las culturas, por lo que pertenecer a un grupo étnico-cultural podría no ser un factor que afecte a la IE (Miao et al., 2020). Así mismo, es interesante conocer si existen diferencias entre los grupos étnico-culturales en cuanto a la inteligencia emocional.

\section{Competencias socioemocionales}

Concibiendo que la IE es una habilidad del individuo, susceptible de desarrollo, la educación debe fomentar su mejora y aplicabilidad para la vida, a través del desarrollo de las competencias socioemocionales como elemento esencial del currículum.

Las competencias socioemocionales son competencias que ayudan a reconocer, procesar y gestionar las emociones de uno mismo y las de los otros (CASEL, 2012; Zych, et al., 2018a). Los componentes básicos para el desarrollo de las competencias socioemocionales incluyen autoconciencia, 
autogestión y motivación, conciencia social y conducta prosocial, y toma de decisiones responsable (Zych, et al., 2018b).

La adquisición óptima de estas competencias socioemocionales puede prevenir numerosos riesgos futuros relacionados con el consumo de sustancias, violencia y depresión (Nasaescu, et al., 2020; Zych, et al., 2020). Los entornos escolares son un lugar idóneo para el entrenamiento continuado de estas competencias asegurando una buena adaptación educativa y social (CASEL, 2003).

Un alto nivel de las competencias socioemocionales se relaciona positivamente con mejores comportamientos sociales, menos conductas problemáticas y menos estrés emocional (Greenberg et al., 2003). A su vez, existe una relación negativa entre las competencias socioemocionales y los problemas personales, sociales y académicos (Durlak et al., 2011; Llorent et al., 2020). Es por ello, que las competencias socioemocionales pueden ser un factor de protección ante situaciones de violencia, como el acoso escolar o ciberacoso (Zych, et al., 2018a).

Existe la necesidad de estudiar las competencias socioemocionales de diversos grupos étnicoculturales para promover la educación inclusiva (Llorent et al., 2016). Las intervenciones que se han realizado sobre el fortalecimiento de las competencias socioemocionales en contextos diversos muestran que se necesitan tener en cuenta las características personales de cada grupo para mejorar sus competencias (Ayotte et al., 2003; Garner et al., 2014). Otro estudio llevado a cabo con 65 niños asiáticoamericanos y latinoamericanos en clases multiculturales desveló que las competencias socioemocionales diferían entre ambos grupos, siendo los niños latinoamericanos los que empleaban con mayor frecuencia un enfoque moral que los niños asiático-americanos. En este mismo estudio también se observó un progreso diferente en las competencias socioemocionales al principio y final del curso escolar. Un ejemplo es la evitación de conflictos, donde los escolares asiático-americanos aumentaron sus puntuaciones, mientras que niños y niñas latinoamericanos las disminuyeron (Petrowski et al., 2009). El estudio de Soriano-Ayala y Osorio-Méndez (2008) evaluó las competencias socioemocionales del alumnado almeriense. Este estudio con una muestra de origen español y latinoamericano mostró diferencias significativas en la habilidad de identificar las propias emociones y sentimientos con respecto a los alumnos de origen magrebí y subsahariano. Los investigadores encontraron diferencias significativas únicamente en la variable autoconciencia.

\section{El presente estudio}

Tal y como se ha descrito, la mejora de la IE y la adquisición de competencias socioemocionales favorece a todo el alumnado del aula a nivel escolar, social y personal. Pero, más aún será necesario para aquellos grupos de alumnos que pueden necesitar una mayor atención como son los grupos étnicosculturales minoritarios. En este sentido, el primer objetivo de este estudio es conocer y analizar la diversidad étnico-cultural que existe en las aulas andaluzas. Como hipótesis de partida, se plantea que existe una presencia heterogénea de diferentes grupos étnicos-culturales en las escuelas de Andalucía. El segundo objetivo fue conocer el nivel de IE percibida y las competencias socioemocionales en diferentes grupos étnico-culturales, así como la relación entre estas variables. En la segunda hipótesis se plantea la existencia de diferencias en los niveles de IE percibida y de competencias socioemocionales en los grupos étnicos-culturales.

\section{Metodología}

\section{Participantes}

La selección de la muestra ha sido realizada por un muestreo aleatorio por conglomerados, teniendo en cuenta las 8 provincias andaluzas, la titularidad del centro (pública o privada) y el número 
de habitantes de los municipios (pequeño < 10,000; mediano entre 10,000 y 100,000; grande > 100,000). Se obtuvo una muestra representativa compuesta por 2,139 discentes de Educación Secundaria Obligatoria de Andalucía (España). El 50.9\% fueron chicas y el 48\% fueron chicos. La edad media de los sujetos fue de 13.79 años $(D T=1.40)$, con un rango de entre 11 y 19 años. La muestra se recogió en los cuatro cursos de la etapa de Educación Secundaria Obligatoria $\left(1^{\circ}=25.3 \% ; 2^{\circ}=25.9 \% ; 3^{\circ}=24.7 \%\right.$ y $\left.4^{\circ}=23.7 \%\right)$.

Se han distinguido cuatro grupos según sus características étnico-culturales. El primer grupo se ha compuesto por adolescentes de etnia gitana $(n=101)$. El segundo grupo está compuesto por inmigrantes de primera generación donde el estudiante tiene nacionalidad extranjera $(n=136)$. El tercer grupo son inmigrantes de segunda generación donde, al menos, uno de los padres tiene nacionalidad extranjera $(n=178)$. Por último, el grupo mayoritario fue compuesto por el alumnado de nacionalidad española y no gitano $(n=1,636)$. Cabe mencionar que 88 alumnos no han informado sobre su grupo étnico-cultural $(4.1 \%)$.

\section{Instrumentos}

El instrumento ha estado compuesto por una serie de cuestiones sociodemográficas (sexo, edad, curso académico, etnia, nacionalidad y nacionalidad del padre y de la madre), la escala de inteligencia emocional percibida y la escala de competencias socioemocionales. Para medir la IE percibida, se ha empleado la versión española de la escala Trait Meta-Mood Scale (TMMS-24; Fernández-Berrocal et al., 2004). El TMMS-24 se responde en una escala de tipo Likert de 5 puntos que oscila de 1 (nada de acuerdo) a 5 (Totalmente de acuerdo). El cuestionario está compuesto por 24 ítems dividido en tres dimensiones con buenos índices de fiabilidad en este estudio: atención emocional, $(\alpha=.93, \Omega=.92)$, comprensión emocional $(\alpha=.93, \Omega=.92)$ y regulación emocional $(\alpha=.90, \Omega=.90)$. El cuestionario en total ha obtenido excelentes resultados en cuanto a su fiabilidad ( $\alpha=.94 ; \Omega=.94)$, y buen ajuste de los datos a la estructura de tres factores $(\mathrm{SB} \chi 2=1,819.68 ; d f=249 ; \mathrm{NFI}=.98 ; \mathrm{NNFI}=.98 ; \mathrm{CFI}=.98$; RMSEA $=.06$, IC 90\% $=.057-.062$ ).

La escala utilizada para medir las Competencias Socioemocionales del alumnado ha sido el Cuestionario de Competencias Socioemocionales (SEC-Q, Social and Emotional Competencies Questionnaire), elaborado y validado por Zych, et al. (2018b). Este cuestionario está compuesto por 16 ítems respondidos en una escala de 5 puntos de tipo Likert, cuyas respuestas varían de 1 (totalmente en desacuerdo) a 5 (totalmente de acuerdo), con unos excelentes índices de fiabilidad $(\alpha=.82 ; \Omega=.82)$. El cuestionario SEC-Q incluye cuatro competencias: autoconciencia $(\alpha=.82 ; \Omega=.71)$, autogestión y motivación $(\alpha=.65 ; \Omega=.67)$, conciencia social y conducta prosocial $(\alpha=.71 ; \Omega=.72)$, y toma de decisiones responsable ( $\alpha=.76 ; \Omega=.76)$. El análisis factorial confirmatorio de la escala mostró un buen ajuste de los datos de este estudio a esta estructura de cuatro factores $(\mathrm{SB} \chi 2=283.30 ; d f=98 ; \mathrm{NFI}=.97$; $\mathrm{NNFI}=.97 ; \mathrm{CFI}=.98 ; \mathrm{RMSEA}=.032, \mathrm{IC} 90 \%=.027-.036)$.

\section{Procedimiento}

Este estudio descriptivo e inferencial se realizó a través de una encuesta aplicada a una muestra representativa de estudiantes de la Educación Secundaria Obligatoria de Andalucía (España). Una vez obtenidos los permisos necesarios tanto por el centro educativo como por las familias del alumnado, se acordaron las visitas de los investigadores para la recogida de datos.

La aplicación del cuestionario se realizó en papel, de forma individual, en el aula de referencia y en horario escolar. Los propios investigadores del estudio informaron, aplicaron y recogiendo los datos directamente del alumnado participante, por lo que se les garantizaba su anonimato en todo momento. La duración de la cumplimentación de la encuesta fue de aproximadamente 30 minutos. 
Este trabajo se desarrolló cumpliendo con los principios éticos nacionales e internacionales incluyendo el marco de la Declaración de Helsinki y la ley de protección de datos. El estudio fue aprobado por el Comité Ético de la Universidad de Córdoba.

\section{Análisis de Datos}

El análisis de datos se ha llevado a cabo con el paquete estadístico SPSS en su versión 23 y FACTOR en su versión 10.3. En primer lugar, se llevaron a cabo los análisis de la fiabilidad calculando el alfa de Cronbach y la omega de McDonald con el software FACTOR. Se han realizado los análisis factoriales confirmatorios con el programa EQS 6.2. Posteriormente, se ha realizado un análisis descriptivo teniendo en cuenta los grupos étnicos-culturales de la muestra, con los porcentajes totales en las diferentes variables sociodemográficas, comparado a través de la prueba de chi-cuadrado y tablas de contingencia.

Finalmente, se han comparado los grupos étnicos-culturales pertenecientes a la mayoría y los grupos minoritarios para descubrir si existen diferencias significativas en IE y competencia socioemocional mediante la prueba t-Student, ANOVA de un factor y ANOVA de Welch, cuyas comparaciones post-hoc se realizaron con la prueba Games-Howell adecuada para las varianzas heterogéneas. Por último, se ha realizado la prueba $d$ de Cohen para calcular el tamaño del efecto teniendo en cuenta los criterios para un tamaño del efecto pequeño $(d=0.20-0.49)$, mediano $(d=0.50-0.79)$, o grande $(\mathrm{d}>0.80)$ según lo que establece Cohen (1988).

\section{Resultados}

En primer lugar, se ha realizado un análisis descriptivo y comparativo de los cuatro grupos étnicos culturales teniendo en cuenta las diferentes variables sociodemográficas (ver Tabla 1). Los datos mostraron que no existen diferencias significativas en función de sexo $\left(\chi^{2}(3,1932)=3.73 p=.29\right)$, pero si en el curso académico en el que se encuentran matriculados los escolares $\left(\chi^{2}(9,1948)=23.87 p=\right.$ .005) con respecto a la distribución de los grupos étnico-culturales. En el análisis de los residuos tipificados se destaca en el grupo de etnia gitana el segundo curso con una mayor presencia (6.6\%; RTC $=2.7)$, a diferencia del cuarto curso con el menor porcentaje $(2.7 \% ; \mathrm{RTC}=2.2)$. Por otro lado, también hay diferencias en el grupo de inmigrantes de primera generación donde en el cuarto curso hay una mayor presencia $(8.6 \%$; RTC $=2.5)$ que en el resto de los grupos.

\section{Tabla 1}

Características sociodemográficas del alumnado en función de los grupos étnico-culturales en las aulas andaluzas

\begin{tabular}{|c|c|c|c|c|}
\hline & $\begin{array}{l}\text { \% Mayoría } \\
(n=1,636)\end{array}$ & $\begin{array}{l}\% \text { Etnia } \\
\text { gitana } \\
(n=101)\end{array}$ & $\begin{array}{c}\% \text { Inmigrantes } 1^{\mathrm{a}} \\
\text { generación } \\
(n=136)\end{array}$ & $\begin{array}{c}\% \text { Inmigrantes } 2^{\mathrm{a}} \\
\text { generación } \\
(n=178)\end{array}$ \\
\hline \multicolumn{5}{|l|}{ Sexo } \\
\hline Hombre & 79.3 & 5.1 & 6.7 & 9.0 \\
\hline Mujer & 82.5 & 3.8 & 5.8 & 8.0 \\
\hline \multicolumn{5}{|l|}{ Curso } \\
\hline Primero & 79.2 & 5.5 & 5.0 & 10.3 \\
\hline Segundo & 81.5 & 6.6 & 4.7 & 7.2 \\
\hline Tercero & 83.2 & 3.0 & 6.6 & 7.2 \\
\hline Cuarto & 79.6 & 2.7 & 8.6 & 9.1 \\
\hline \multicolumn{5}{|l|}{ Provincia } \\
\hline Almería & 58.6 & 4.4 & 20.4 & 16.6 \\
\hline
\end{tabular}




\begin{tabular}{|c|c|c|c|c|}
\hline Cádiz & 88.0 & 4.1 & 2.5 & 5.4 \\
\hline Córdoba & 90.3 & 2.4 & 3.0 & 4.2 \\
\hline Granada & 82.2 & 4.5 & 4.1 & 9.3 \\
\hline Huelva & 87.8 & 1.0 & 6.1 & 5.1 \\
\hline Jaén & 80.8 & 5.6 & 10.2 & 3.4 \\
\hline Málaga & 74.0 & 3.8 & 7.6 & 14.6 \\
\hline Sevilla & 83.3 & 6.6 & 2.9 & 7.2 \\
\hline \multicolumn{5}{|l|}{ Tamaño localidad } \\
\hline Pequeño & 90.8 & 5.6 & 0.4 & 3.2 \\
\hline Mediano & 81.6 & 4.2 & 6.6 & 7.6 \\
\hline Grande & 76.9 & 4.6 & 7.8 & 10.8 \\
\hline \multicolumn{5}{|l|}{$\begin{array}{l}\text { Titularidad } \\
\text { centro }\end{array}$} \\
\hline Pública & 80.4 & 5.0 & 6.8 & 7.9 \\
\hline Privada & 80.9 & 4.4 & 6.3 & 8.4 \\
\hline Total & 79.8 & 4.9 & 6.6 & 8.7 \\
\hline
\end{tabular}

El análisis realizado entre las ocho provincias andaluzas demostró la existencia de diferencias significativas entre los grupos $\left(\chi^{2}(21,1861)=146.58, p=.000\right)$. El análisis de los residuos tipificados mostró mayor presencia del grupo mayoritario en las escuelas cordobesas $(90.3 \%$; $\mathrm{RTC}=3.3)$ respecto al resto, siendo la escuela almeriense dónde menos porcentaje del grupo mayoritario hubo (58.6\%; RTC $=7.8$ ). En cuanto al grupo de etnia gitana, el mayor porcentaje respecto a las demás escuelas se encontró en los centros educativos de Sevilla $(6.6 \%$; RTC $=2.3)$. Las aulas de Almería tuvieron mayores porcentajes en el grupo de inmigrantes de primera generación $(20.4 \%$; $\mathrm{RTC}=8.2)$ con respecto al resto. La menor presencia de estudiantes inmigrantes de primera generación se encontró en las escuelas de Cádiz $(2.5 \%$; RTC $=2.6)$. Los residuos tipificados corregidos mostraron mayor presencia del grupo de inmigrantes de segunda generación en los centros de la provincia de Almería $(16.6 \%$; RTC $=4.0)$ y menor presencia en los centros de Jaén $(3.4 \%$; RTC $=2.6)$ en comparación con los centros educativos de las demás provincias.

Igualmente, se han encontrado diferencias significativas entre los diferentes grupos en relación con el tamaño de la localidad $\left(\chi^{2}(6,1861)=36.92, p=.000\right)$. El análisis de los residuos tipificados mostró mayor presencia del grupo mayoritario en las localidades con un tamaño pequeño $(90.8 \%$; RTC $=4.4)$ en comparación con las demás, siendo las localidades de tamaño grande dónde hubo menor porcentaje del grupo mayoritario $(76.9 \%$; RTC $=3.9)$. En el caso del grupo de estudiantes de etnia gitana, los residuos tipificados corregidos no mostraron mayores o menores porcentajes según el tamaño de la localidad en la que se sitúa el centro. Para los inmigrantes de primera generación, es destacable una menor presencia de éstos en localidades de tamaño pequeño $(0.4 \% ; \mathrm{RTC}=4.1)$ con respecto a las demás. Por último, en el caso de los inmigrantes de segunda generación, hubo una mayor presencia de este grupo en las localidades de tamaño pequeño $(3.2 \%$; RTC $=3.3)$ en comparación con localidades medianas y grandes.

También se han encontrado diferencias significativas entre los grupos según la titularidad del centro educativo $\left(\chi^{2}(3,1949)=12.37 p=.006\right)$. El análisis de los residuos tipificados mostró mayor presencia del grupo mayoritario en centros de titularidad privada $(83.0 \%$; RTC $=1.2)$ con respecto a centros públicos $(80.4 \% ; \mathrm{RTC}=1.2)$. En el caso del grupo de estudiantes de etnia gitana, se encontró un mayor porcentaje en los centros de titularidad pública $(5.0 \%$; RTC $=2.5)$ en contraste con los centros privados $(2.1 \%$; RTC $=2.5)$. Los estudiantes pertenecientes al grupo de inmigrantes de primera 
generación tuvieron una mayor presencia en los centros públicos $(6.8 \%$; RTC $=1.9)$ en comparación con los centros privados $(4.2 \%$; RTC $=1.9)$. Por último, en el caso de los inmigrantes de segunda generación, los residuos tipificados corregidos no mostraron un mayor o menor porcentaje según la titularidad del centro.

\section{Inteligencia emocional y competencias socioemocionales en diferentes grupos étnico-culturales}

En la Tabla 2, se muestran las puntuaciones medias del alumnado de educación secundaria en los niveles de $I E$, y competencias socioemocionales. Las puntuaciones totales en ambas escalas fueron excelentes ya que, superan la media de 3 puntos, siguiendo las indicaciones del baremo EspinozaVenegas et al. (2015). Si se comparan las dimensiones de las dos escalas, se observa como autoconciencia y autogestión y motivación tuvieron una media elevada que alcanza los 4 puntos (de acuerdo) de la escala Likert. En contraposición, la media más baja se destacó en atención emocional, aunque se encuentran en niveles adecuados.

\section{Tabla 2}

Medias y desviaciones típicas en nivel de inteligencia emocional y competencias socioemocionales

\begin{tabular}{lcc}
\hline Inteligencia emocional & $M$ & $D T$ \\
\hline Atención emocional & 3.12 & 1.02 \\
\hline Comprensión emocional & 3.31 & 0.98 \\
\hline Regulación emocional & 3.48 & 0.96 \\
\hline Total & 3.31 & 0.80 \\
\hline Autoconciencia & $M$ & $D T$ \\
\hline Autogestión y motivación & 4.00 & 0.69 \\
\hline Conciencia social y conducta prosocial & 4.05 & 0.80 \\
\hline Toma de decisiones responsable & 3.55 & 0.56 \\
\hline Total & 3.92 & 0.92 \\
\hline
\end{tabular}

Tras el análisis global de las puntuaciones en $I E$ y en las competencias socioemocionales en la muestra, se realizaron las comparaciones entre los grupos étnico-culturales. Se hicieron dos grupos, el mayoritario con 1636 (76.5\%) sujetos con nacionalidad española que no sean de etnia gitana y el grupo minoritario compuesto por $415(19.4 \%)$ sujetos de etnia gitana, y los inmigrantes de $1^{\circ}$ y $2^{\circ}$ generación. También se hicieron comparaciones incluyendo los cuatro grupos étnico-culturales por separado.

\section{Tabla 3}

Comparación de la puntuación en inteligencia emocional y competencias socioemocionales entre los grupos étnico-culturales

\begin{tabular}{lccccccc} 
& Mayoría & Minoría & & $\begin{array}{c}\text { Inmigrantes } \\
\text { de primera } \\
\text { generación }\end{array}$ & $\begin{array}{c}\text { Inmigrantes } \\
\text { de segunda } \\
\text { generación }\end{array}$ & Gitanos \\
\hline & $\mathrm{M}(D T)$ & $\mathrm{M}(D T)$ & $t$ & $\mathrm{M}(D T)$ & $\mathrm{M}(D T)$ & $\mathrm{M}(D T)$ & $\mathrm{F}$ \\
\hline $\begin{array}{l}\text { Inteligencia } \\
\text { Emocional }\end{array}$ & & & & & & & \\
\hline $\begin{array}{l}\text { Atención } \\
\text { emocional }\end{array}$ & $3.14(1)$ & 3.07 & 1.22 & $2.98(1.07)$ & $3.04(1.05)$ & 3.22 & 1.521 \\
\hline $\begin{array}{l}\text { Comprensión } \\
\text { emocional }\end{array}$ & 3.31 & 3.33 & -0.29 & $3.25(1.1)$ & $3.33(1.05)$ & 3.42 & $0.414^{\mathrm{a}}$ \\
\hline
\end{tabular}




\begin{tabular}{|c|c|c|c|c|c|c|c|}
\hline $\begin{array}{l}\text { Regulación } \\
\text { emocional }\end{array}$ & $\begin{array}{c}3.49 \\
(0.93)\end{array}$ & $\begin{array}{c}3.47 \\
(1.06)\end{array}$ & 0.39 & $3.40(1.03)$ & $3.48(1.02)$ & $\begin{array}{c}3.53 \\
(1.16) \\
\end{array}$ & $0.318^{\mathrm{a}}$ \\
\hline Total & $\begin{array}{l}3.31 \\
(0.77) \\
\end{array}$ & $\begin{array}{c}3.33 \\
(0.90) \\
\end{array}$ & -0.37 & $3.22(0.83)$ & $3.32(.89)$ & $\begin{array}{c}3.46 \\
(0.99) \\
\end{array}$ & 1.089 \\
\hline \multicolumn{8}{|l|}{$\begin{array}{l}\text { Competencias } \\
\text { Socioemocionales }\end{array}$} \\
\hline Autoconciencia & $\begin{array}{r}4.03 \\
(0.65) \\
\end{array}$ & $\begin{array}{r}3.91 \\
(0.81) \\
\end{array}$ & $2.75^{* *}$ & $3.83(0.83)$ & $3.98(0.76)$ & $\begin{array}{c}3.87 \\
(0.86) \\
\end{array}$ & $3.194^{\mathrm{a} *}$ \\
\hline $\begin{array}{l}\text { Autogestión y } \\
\text { motivación }\end{array}$ & $\begin{array}{c}4.06 \\
(0.78)\end{array}$ & $\begin{array}{c}4.02 \\
(0.85)\end{array}$ & 0.78 & $4.07(0.84)$ & $3.98(0.81)$ & $\begin{array}{r}4.04 \\
(0.92) \\
\end{array}$ & 0.557 \\
\hline $\begin{array}{l}\text { Conciencia } \\
\text { social y } \\
\text { conducta } \\
\text { prosocial }\end{array}$ & $\begin{array}{c}3.97 \\
(0.54)\end{array}$ & $\begin{array}{c}3.94 \\
(0.65)\end{array}$ & 1.31 & $3.86(0.65)$ & $3.98(0.58)$ & $\begin{array}{c}3.91 \\
(0.74)\end{array}$ & $1.346^{\mathrm{a}}$ \\
\hline $\begin{array}{l}\text { Toma de } \\
\text { decisiones } \\
\text { responsable }\end{array}$ & $\begin{array}{c}3.57 \\
(0.91)\end{array}$ & $\begin{array}{c}3.48 \\
(0.95)\end{array}$ & 1.9 & $3.55(0.88)$ & $3.48(0.95)$ & $\begin{array}{c}3.38 \\
(1.05)\end{array}$ & 1.843 \\
\hline Total & $\begin{array}{c}3.94 \\
(0.46)\end{array}$ & $\begin{array}{c}3.85 \\
(0.56)\end{array}$ & $2.62 * *$ & $3.84(0.53)$ & $3.89(0.51)$ & $\begin{array}{c}3.81 \\
(0.66)\end{array}$ & 2.442 \\
\hline
\end{tabular}

Nota. ${ }^{a}$ ANOVA de Welch; ${ }^{*} p<.05 ; * * p<.01$

Como reflejan las comparaciones de medias de la Tabla 3, el grupo mayoritario y minoritario superaron la puntuación media de 3 puntos en la $I E$, pero en ninguna de las dimensiones superaron una puntuación de 4, siendo estos resultados adecuados en los tres factores de la $I E$ (Espinoza-Venegas, et al., 2015). El análisis comparativo en $I E$ entre el grupo mayoritario y minoritario mostró que no existían diferencias significativas entre ambos grupos.

Por otro lado, los resultados de la comparación de las competencias socioemocionales (Tabla 3), mostraron que el grupo mayoritario se percibió con mejor autoconciencia que el grupo minoritario. Igualmente, el grupo mayoritario se autopercibió de manera más favorable en las competencias socioemocionales en su conjunto en comparación con el grupo minoritario. La prueba $d$ de Cohen mostró que el tamaño del efecto era pequeño tanto con respecto a la diferencia en la autoconciencia $(d=0.18$; $95 \% \mathrm{CI}=0.07-2.9)$ como en el total de las competencias socioemocionales autopercibidas $(d=0.18$; $95 \% \mathrm{CI}=0.07-2.9$ ).

Teniendo en cuenta la comparación de los cuatro grupos étnico-culturales (el mayoritario, inmigrantes de primera generación, inmigrantes de segunda generación y alumnado de etnia gitana), en cuanto a la inteligencia emocional, es destacable que las puntuaciones más bajas se dieron en la dimensión atención emocional y las puntuaciones más elevadas en el factor regulación emocional en todos los grupos. No se han encontrado diferencias significativas en el nivel de $I E$ autopercibida por los cuatro grupos étnico-culturales ni en la puntuación total ni en sus subescalas.

Con respecto a las competencias socioemocionales, se mostraron diferencias significativas entre los grupos étnico-culturales en el factor de autoconciencia, pero sin que se reflejasen en el análisis post hoc de autoconciencia ( $p>.05$ en todas las comparaciones). La prueba $d$ de Cohen sí mostró que el grupo mayoritario se autopercibió con mejor autoconciencia que los estudiantes de etnia gitana $(d=0.24 ; 95 \%$ $\mathrm{CI}=0.04-0.44)$ y los inmigrantes de primera generación $(d=0.29 ; 95 \% \mathrm{CI}=0.11-0.47)$. 


\section{Discusión}

Este trabajo estudia la diversidad étnico-cultural, la IE y las competencias socioemocionales en los estudiantes de Educación Secundaria Obligatoria de Andalucía (España). La importancia de esta investigación reside en la necesidad de constatar la presencia de diferentes grupos étnico-culturales y aportar conocimiento científico para el desarrollo de una educación inclusiva, valorando la IE y las competencias socioemocionales de todos los estudiantes.

En primer lugar, atendiendo a la distribución de los grupos étnico-culturales según el curso académico. En el estudio se evidencia como en el grupo de etnia gitana va disminuyendo su presencia a medida que llega el final de la escolarización obligatoria, del mismo modo que se muestra en el informe FSG (2013). Si bien, en el grupo de inmigrantes de primera generación, ocurren todo lo contrario, es en el cuarto curso donde hay una mayor presencia de esta minoría.

En relación con la escolarización por provincias, los resultados respecto a la mayor presencia de los inmigrantes de primera y segunda generación en los centros educativos de Almería son similares a los datos sobre la población general del Instituto de Estadística y Cartografía de Andalucía (IECA, 2014). Asimismo, se ha encontrado que la mayor presencia del grupo mayoritario se da en las escuelas cordobesas. Los discentes gozan de una mayor posibilidad de convivencia intercultural en las aulas de la provincia de Almería, a diferencia de las escuelas de la provincia de Córdoba, donde el alumnado es menos diverso desde el punto de vista étnico-cultural. Por tanto, se comprueba que los grupos minoritarios no solo están presentes en la sociedad, sino que también están presentes en las aulas de las escuelas andaluzas. Especialmente interesante intuir que la población inmigrante con más tradición y estabilidad laboral en un territorio como Almería también parece más asentada desde el punto de vista familiar con la presencia de sus hijos en las escuelas. Esto supone la responsabilidad y la oportunidad de fomentar desde la escuela la inclusión educativa y también social de esta población.

En cuanto al grupo de etnia gitana, Andalucía es la comunidad autónoma donde tiene más presencia (Fundación de Secretariado Gitano, 2009; Laparra y García, 2011). Un estudio realizado en 2010 estimó que las provincias de Granada y Sevilla destacaban por tener más viviendas pertenecientes a la población gitana (Pita-López y Pedregal-Mateos, 2011). En el presente estudio también aparece la provincia de Sevilla con el mayor porcentaje de estudiantes de Educación Secundaria Obligatoria perteneciente a la etnia gitana y Huelva como la provincia con menos alumnado de etnia gitana. Estos datos aportan un mayor conocimiento de la escolarización y presencia real en las aulas de los chicos y chicas de etnia gitana en Andalucía. Otro dato relevante que se observa es la semejanza que existe entre el alumnado y la población en cada provincia. Esto quiere decir que todos los grupos étnicos-culturales están escolarizados y que, además, estuvieron presentes en el momento de la recogida de datos, aportando una visión positiva a los esfuerzos políticos y escolares de inclusión educativa.

Atendiendo al tamaño de la localidad donde se sitúa el centro, esta investigación muestra que el alumnado inmigrante se concentra en escuelas situadas en zonas urbanas de gran tamaño (más de 100,000 habitantes). Por el contrario, el grupo mayoritario representa un mayor porcentaje en localidades de tamaño pequeño (menos de 10,000 habitantes) coincidiendo con los datos estadísticos ofrecidos por el Ministerio de Educación, Política Social y Deporte (2009). Esto podría entenderse desde el supuesto de que posiblemente los hijos de estos inmigrantes no están escolarizados en zonas rurales o bien aún no acompañan a los adultos y se han quedado en sus países de origen.

En el caso de la titularidad del centro, tal y como se ha detectado en el presente estudio, la población gitana toma la opción de escolarizar a sus hijos en centros de titularidad pública de forma más acusada que el resto de la población. De manera que el grupo mayoritario es más representado en centros de titularidad privada. Un estudio llevado a cabo por la Fundación de Secretariado Gitano (2013) con una muestra de 1,604 personas de etnia gitana que cursaban la Educación Secundaria Obligatoria, 
encontró datos similares a los hallados en la presente investigación. Según dicho estudio, el 80.4\% de chicos y el $75.1 \%$ de chicas de etnia gitana estaban matriculados en centros de titularidad pública, mientras que un $15.5 \%$ de chicos y un $20.7 \%$ de chicas de etnia gitana estudiaban en centros privadosconcertados. En cuanto a los estudiantes inmigrantes de primera generación, los resultados de este estudio han mostrado mayor presencia en centros públicos, al igual que el alumnado de etnia gitana. Existen datos similares encontrados por el Ministerio de Educación y Formación profesional (2018), pues la distribución de los estudiantes extranjeros por titularidad del centro en el curso 2013-2014 muestra mayores porcentajes de este alumnado en los centros públicos andaluces. Por el contrario, los centros privados destacan por el mayor porcentaje del alumnado del grupo mayoritario. Este hecho no debería conducir a estereotipos de nivel socioeconómico o cultural, pues en este presente estudio no se ha tenido en cuenta dicha valoración. Si bien, la diversidad en las aulas es un factor que enriquece al alumnado (Byrd, 2015), por tanto, tendría que haber más presencia de grupos étnico-culturales también en los centros privados. Según los datos obtenidos en el estudio, se confirma la primera hipótesis de la investigación en donde se planteaba que existía una presencia heterogénea de diferentes grupos étnicosculturales en las escuelas de Andalucía.

Entrando en los niveles de la IE en los grupos étnicos-culturales, no se han hallado diferencias significativas entre el grupo mayoritario y minoritario en ninguno de los componentes de la IE percibida y, tampoco cuando se han comparado los cuatro grupos étnico-culturales (mayoría, inmigrantes de primera generación, inmigrantes de segunda generación y etnia gitana). En vista de los datos obtenidos, no se puede confirmar la segunda hipótesis, ni ratificar los estudios anteriores (Dewi et al., 2018; Martines et al., 2006). El hecho de no hallar diferencias en la IE percibida entre los grupos étnicoculturales estudiados revela que el alumnado andaluz, independientemente de su origen étnico-cultural, se perciben con una atención, comprensión y regulación emocional similar. Estos datos pueden evidenciar que el grupo étnico-cultural no está relacionado con la IE o que todas las culturas valoran la IE y por ello no hay esas diferencias significativas (Miao et al., 2020).

En relación con el nivel de competencias socioemocionales, los escolares andaluces obtienen buenas puntuaciones. Los datos han confirmado, en parte, la hipótesis que planteaba que habría diferencias significativas en las competencias socioemocionales según los grupos étnicos-culturales. También se confirman los datos de Soriano-Ayala y Osorio-Méndez (2008) donde se encuentran diferencias significativas en la dimensión autoconciencia, de igual manera que en este estudio. La diversidad étnico-cultural puede verse afectada por las situaciones socioeconómicas individuales, donde las minorías pueden ser más vulnerables, y sus relaciones sociales más limitadas. Por tanto, la escuela debe afrontar el reto de facilitar una formación en competencias socioemocionales que asegure la igualdad de oportunidades. Resulta interesante el contraste entre la no diferencia de las habilidades personales de inteligencia emocional y las competencias, en este caso socioemocionales. Ya que las competencias se suelen fomentar desde la escuela y tiene potencial de crecimiento, como el resto de las competencias.

Esta investigación presenta como punto fuerte una muestra amplia y representativa, lo que permite tener una información extensa de la realidad escolar andaluza. En cuanto a las limitaciones, éstas se derivan del uso de autoinformes, pues aun garantizando el anonimato, puede existir el sesgo de deseabilidad social. Como línea futura de investigación, se plantea realizar una intervención educativa basada en la inclusión que puede fomentar las competencias socioemocionales y, al mismo tiempo, favorecer la convivencia del aula (Ortega, 2015), el bienestar y seguridad en la escuela, y el reconocimiento de las propias emociones y las de los demás.

Las competencias socioemocionales tienen un nivel distinto, según el grupo étnico-cultural, que podría deberse a la formación personal y escolar. De esta forma, queda claro que la escuela, como institución educativa, podría tener capacidad para potenciar de la misma manera las competencias 
socioemocionales, como las demás competencias, entre todo el alumnado, facilitando la atención a la diversidad, la igualdad de oportunidades y, por tanto, la educación inclusiva.

\section{Agradecimientos}

Este trabajo se ha llevado a cabo en el marco del Proyecto de investigación titulado "Adicción a las nuevas tecnologías: el papel de las cibercompetencias emocionales e inteligencia emocional" BIL/14/S2/163 dado por la Fundación MAPFRE y por el proyecto financiado por Ministerio de Economía y Competitividad dentro del Programa Nacional de Investigación I+D+I 2015 "E-inteligencia: riesgos y oportunidades de las competencias emocionales expresadas en la red" (PSI2015-64114-R) ambos otorgados a la segunda autora y al equipo investigación.

\section{Referencias}

Aguaded, E. M. (2006). La educación de competencias básicas para el desarrollo de la ciudadanía intercultural en la ESO. Actas Del V Congreso Internacional "Educación y Sociedad: Retos Del s. XXI, 21, 1-8. Universidad de Granada.

Ayotte, V., Saucier, J., Bowen, F., y Laurende, M. (2003). Teaching Multiethnic Urban Adolescents How to Enhance Their Competencies: Effects of a middle school primary prevention program on adaptation. Journal of Primary Prevention. 24(1), 7-23.

Beltrán-Catalán, M., Zych, I., Ortega-Ruiz, R., y Llorent, V. J. (2018). Victimisation through bullying and cyberbullying: Emotional intelligence, severity of victimisation and technology use in different types of victims. Psicothema, 30(2), 183-188. https://doi.org/10.7334/psicothema2017.313

Berking, M., Orth, U., Wupperman, P., Meier, L. L., y Caspar, F. (2008). Prospective Effects of EmotionRegulation Skills on Emotional Adjustment. Journal of Counseling Psychology, 55(4), 485-494. https://doi.org/10.1037/a0013589

Byrd, C. M. (2015). The associations of intergroup interactions and school racial socialization with academic motivation. Journal of Educational Research, 108(1), 10-21. https://doi.org/10.1080/00220671.2013.831803

Checa, J. C., y Monserrat, M. (2015). La integración social de los hijos de inmigrantes africanos, europeos del este y latinoamericanos: Un estudio de caso en España. Universitas Psychologica, 14(2), 475-486. https://doi.org/10.11144/Javeriana.upsy14-2.lish

Ciarrochi, J., Chan, A., Caputi, P., y Roberts, R. (2001). Measuring emotional intelligence (EI). In J. V. Ciarrochi, J. P. Forgas, y J. D. Mayer (Eds.), Emotional Intelligence in Everyday Life (pp. 25-44). Psychology Press.

Cohen, J. (1988). Statistical Power Analysis for the Behavioral Sciences (2a Ed.). Lawrence Erlbaum Associates.

Collaborative for Academic, Social and Emotional Learning (2003). Safe and sound: An educational leader's guide to evidence-based social and emotional learning programs. Autor.

Collaborative for Academic, Social and Emotional Learning (2012). 2013 CASEL guide: Effective social and emotional learning programs-Preschool and elementary school edition. Autor.

Dewi, Z. L., Halim, M. S., y Derksen, J. (2018). Emotional intelligence competences of three different ethnic groups in Indonesia. Asian Ethnicity, 19(1), 36-58. https://doi.org/10.1080/14631369.2017.1310615

Espinoza-Venegas, M., Sanhueza-Alvarado, O., Ramírez-Elizondo, N., y Sáez-Carrillo, K. (2015). Validación de constructo y confiabilidad de la escala de inteligencia emocional en estudiantes de enfermería. Revista latino-americana de enfermagem, 23(1), 139-147

Durlak, J. A., Weissberg, R. P., Dymnicki, A. B., Taylor, R. D., y Schellinger, K. B. (2011). The Impact 
of Enhancing Students' Social and Emotional Learning: A Meta-Analysis of School-Based Universal Interventions. Child Development, 82(1), 405-432. https://doi.org/10.1111/j.14678624.2010.01564.x

Fernández-Berrocal, P., Extremera, N., y Ramos, N. (2004). Validity and reliability of the Spanish modified version of the Trait Meta-Mood Scale. Psychological Reports, 94, 751-755.

Fernández-Berrocal, Pablo, y Ramos, N. (2005). Evaluando la inteligencia emocional. In P. FernándezBerrocal y N. Ramos-Díaz (Eds.), Corazones inteligentes (pp. 35-38). Kairós. https://bit.ly/3ulEGrS

Fundación de Secretariado Gitano, F. (2009). Mapa sobre Vivienda y Comunidad Gitana en España, 2007: conclusiones, recomendaciones y propuestas. Revista Bimestral de La Fundación de Secretariado Gitano, 3, 33-48.

Fundación de Secretariado Gitano [FSG]. (2013). El alumnado gitano en secundaria: un estudio comparado. Ministerio de Educación, Cultura y Deporte.

Garner, P. W., Mahatmya, D., Brown, E. L., y Vesely, C. K. (2014). Promoting Desirable Outcomes Among Culturally and Ethnically Diverse Children in Social Emotional Learning Programs: A Multilevel Heuristic Model. Educational Psychology Review, 26(1), 165-189. https://doi.org/10.1007/s10648-014-9253-7

Garreta, J., y Llevot, N. (2007). Los gitanos en España: mercado de trabajo y educación: crónica de un desencuentro. Educacion y Diversidad: Revista Inter-Universitaria de Investigación Sobre Discapacidad e Interculturalidad, 1, 257-278.

Gohm, C. L., y Clore, G. L. (2002). Four latent traits of emotional experience and their involvement in well-being, coping, and attributional style. Cognition and Emotion, 16(4), 495-518. https://doi.org/10.1080/02699930143000374

Greenberg, M. T., Weissberg, R. P., O’Brien, M. U., Zins, J. E., Fredericks, L., Resnik, H., y Elias, M. J. (2003). Enhancing school-based prevention and youth development through coordinated social, emotional, and academic learning. American Psychologist, 58(6-7), 466-474. https://doi.org/10.1037/0003-066X.58.6-7.466

Instituto Nacional de Estadística [INE]. (2007). Encuesta Nacional de Inmigrantes. Resultados por comunidad autónoma. Año 2007. Instituto Nacional de Estadística. Recuperado de: https://bit.ly/2yS234s

Instituto Nacional de Estadística [INE]. (2020). Cifras de Población. Provisionales a 1 de Julio de 2019. In Instituto Nacional de Estadística (Vol. 80, pp. 1-11). Recuperado de: https://www.ine.es/prensa/cp_j2019_p.pdf

Laparra, M., y García, Á. (2011). Una comunidad gitana de tamaño y perfiles todavía imprecisos. En M. Lamparra, (Cood.), Diagnóstico social de la comunidad gitana en España: Un análisis contrastado de la Encuesta del CIS a Hogares de Población Gitana 2007 (pp. 27-34). Ministerio de Sanidad, política social e igualdad.

Llorent-Bedmar, V., y Terrón-Caro, M. T. (2013). La inmigración marroquí en España: Género y educación. Estudios Sobre Educación, 24, 37-59.

Llorent, V. J., y González-Gómez, A. L. (2020). Literacy competence and social and emotional competencies in Primary Education. Individual and ethnic-cultural factors Competencia en comunicación lingüística y competencias. Revista Espacios, 41(6), 1-8.

Llorent, V. J., González-Gómez, A. L., Farrington, D. P., y Zych, I. (2020). Social and emotional competencies and empathy as predictors of literacy competence. Psicothema, 32(1), 47-53. https://doi.org/10.7334/psicothema2019.106

Llorent, V. J., Ortega-Ruiz, R., y Zych, I. (2016). Bullying and cyberbullying in minorities: Are they more vulnerable than the majority group? Frontiers in Psychology, 7, 1-9. 
https://doi.org/10.3389/fpsyg.2016.01507

Martines, D., Fernández-Berrocal, P., y Extremera, N. (2006). Ethnic group differences in perceived emotional intelligence within the United States and Mexico. Ansiedad y Estrés, 12(2), 317-327.

Mayer, J. D., y Salovey, P. (1997). What is emotional intelligence? In P. E. Salovey y D. Sluyter (Eds.), Emotional development and emotional intelligence: Educational implications. (pp. 3-31). Basic Books.

Mayer, J. D., Salovey, P., y Caruso, D. R. (2004). Emotional intelligence: Theory, findings, and implications. Psychological Inquiry, 15(3), 197-215. https://doi.org/10.1207/s15327965pli1503_02

Miao, C., Humphrey, R. H., y Qian, S. (2020). The cross-cultural moderators of the influence of emotional intelligence on organizational citizenship behavior and counterproductive work behavior. Human Resource Development Quarterly, 31(2), 213-233. https://doi.org/10.1002/hrdq.21385

Ministerio de Educación y Formación profesional. (2018). Datos y cifras: Curso escolar 2018/2019. Educación. Ministerio de Educación Cultura y Deporte.

Nasaescu, E., Ortega-Ruiz, R., Llorent, V. J., y Zych, I. (2020). Conductas antisociales en niños y adolescentes: Un estudio descriptivo. Psychology, Society, \& Education, 12(3), 201-213. https://doi.org/10.25115/psye.v10i1.2876

Ortega-Ruiz, R. (2015). Convivencia y ciberconvivencia. Un modelo educativo para la prevención del acoso y el ciberacoso escolar. Antonio Machado.

Petrowski, K., Herold, U., Joraschky, P., von Wyl, A., y Cierpka, M. (2009). The specificity and the development of social-emotional competence in a multi-ethnic-classroom. Child and Adolescent Psychiatry and Mental Health, 3(16) 1-10. https://doi.org/10.1186/1753-2000-3-16

Pita-López, M. F., y Pedregal-Mateos, B. (2011). Dinámica y estados de la población. In M. F. PitaLópez y B. Pedregal-Mateos (Eds.), Tercer Informe de Desarrollo Territorial de Andalucía IDTA 2010 (pp. 223-249).

Portes, A., Aparicio, R., y Haller, W. (2009). La segunda generación en Madrid : un estudio longitudinal. Área: Demografía, Población y Migraciones Internacionales, 67, 1-10.

Portes, A., y Rumbaut, R. G. (2001). Legacies: The story of the immigrant second generation. Berkeley. University of California Press.

Ramos, N. S., Fernández-Berrocal, P., y Extremera, N. (2007). Perceived emotional intelligence facilitates cognitive-emotional processes of adaptation to an acute stressor. Cognition and Emotion, 21(4), 758-772. https://doi.org/10.1080/02699930600845846

Rodríguez-Hidalgo, A. J., Ortega-Ruiz, R., y Zych, I. (2014). Peer victimization and ethnic-cultural peer victimization: Self-esteem and school relations between different cultural groups of students in Andalusia, Spain. Revista de Psicodidactica, 19(1), 191-210. https://doi.org/10.1387/RevPsicodidact.7909

Salovey, Peter, y Grewal, D. (2005). The science of emotional intelligence. Current Directions in Psychological Science, 14(6), 281-285. https://doi.org/10.1111/j.0963-7214.2005.00381.x

Salovey, Peter, Mayer, J. D., Goldman, S., Turvey, C., y Palfai, T. (1995). Emotional attention, clarity, and repair: Exploring emotional intelligence using the Trait Meta-Mood Scale. In J. W. Pennebaker (Ed.), Emotion, Disclosure, \& Health (pp. 125-151). American Psychological Association.

Salovey, Peter, Stroud, L. R., Woolery, A., y Epel, E. S. (2002). Perceived emotional intelligence, stress reactivity, and symptom reports: Further explorations using the trait meta-mood scale. Psychology and Health, 17(5), 611-627. https://doi.org/10.1080/08870440290025812

Sánchez-Álvarez, N., Extremera, N., y Fernández-Berrocal, P. (2015). Maintaining life satisfaction in adolescence: Affective mediators of the influence of perceived emotional intelligence on overall life satisfaction judgments in a two-year longitudinal study. Frontiers in Psychology, 6(1892), 1-9. https://doi.org/10.3389/fpsyg.2015.01892 
Soriano-Ayala, E., y Osorio-Méndez, M. (2008). Competencias emocionales del alumnado "autóctono" e inmigrante de Educación Secundaria. Bordón. Revista de Pedagogía, 60(1), 129-148.

Zych, I., Beltrán-Catalán, M., Ortega-Ruiz, R., y Llorent, V. J. (2018a). Social and Emotional Competencies in Adolescents Involved in Different Bullying and Cyberbullying Roles. Revista de Psicodidactica, 23(2), 86-93. https://doi.org/10.1016/j.psicod.2017.12.001

Zych, I., Ortega-Ruiz, R., Muñoz-Morales, R., y Llorent, V. J. (2018b). Dimensions and psychometric properties of the social and emotional competencies questionnaire (SEC-Q) in youth and adolescents. Revista Latinoamericana de Psicologia, 50(2), 98-106. https://doi.org/10.14349/rlp.2018.v50.n2.3

Zych, I., Rodríguez-Ruiz, J., Marín-López, I., y Llorent, V. J. (2020). Longitudinal stability and change in adolescent substance use: A latent transition analysis. Children and Youth Services Review, 112, 104933. https://doi.org/10.1016/j.childyouth.2020.104933 